\title{
Complicated Engineering Issues about Electrospraying - A Vivid Lesson for Engineering Education in Higher School
}

\author{
Yong-Hui WU ${ }^{1, \text { a,** }}$, Yu-Dan HUANG ${ }^{2}$, Hui-Qi SUN², Yu-Xuan QIN², Meng-Xue ZHOU², Deng-Guang YU2, b,* \\ ${ }^{1}$ The Department of Mechanical Engineering, Guangxi Technological College of Machinery and Electricity, \\ Nanning 530007, China \\ ${ }^{2}$ School of Materials Science \& Engineering, University of Shanghai for Science and Technology \\ Shanghai 200093, China \\ aahuiwyh@163.com, bydg017@usst.edu.cn
}

\begin{abstract}
The capability of finding and resolving complicated engineering issues is very important for the college students to begin their career. In high school, teachers should take advantage of advanced technologies in two ways. One is to transfer the knowledge and practice experiences to the students and the other is to share with them how to find and resolve the complicated issues. In this paper, with electrospraying as an objective, several complicated issues are put forward. Electrospraying is a simple but very complicated engineering process. When it is exploited to create medicated composite for drug sustained release, complicated issues occur not only at the selections of raw materials, suitable working processes and key parameters, but also at the systematic designs of final functional nanomaterials and smooth implementation of working processes. Advanced technologies can be vivid teaching materials for the students to learn how to refine and resolve complex engineering issues, and thus to foster their practice interests, to improve their innovative and engineering capabilities.
\end{abstract}

Keywords-Complicated engineering issue; Electrospraying; Engineering education; Innovative practices; College student; Higher school

\section{INTRODUCTION}

During the four-year study in Chinese university, engineer practice is one of the most important lessons for students majoring in natural science. Numerous innovative projects have been applied and also approved each year in the key universities such as " 985 " and " 211 ". The evaluations of these projects should not only consider if the students have experienced the industrial or scientific practices, but also view the students' capability of putting forward or resolving a complicated engineering issue as an important factor. The ideal situation is that the students can effectively combine their practice experiences with their knowledge learned at classroom, which should promote their fast growth in engineering career.
Certainly, the teachers should instruct the students how to refine complicated engineering issues from their practices, and in turn exploit these issues as vivid teaching materials for fostering their innovation capability.

From a broad meaning, engineering is quite different from science although both of them belong to natural science disciplines. Scientists are always trying their best to understand our world. In sharp contrast, engineers are always trying to create things that do not exist in nature and to solve problem in routine life. To embody an invention the engineer must put his idea in concrete terms, and design something that people can use [1]. The formation of idea, the treated terms, and also the useful products are all associated with a series of complicated engineering issues. Correspondingly, the key points in engineering education in higher education should, on one hand, focus on the applications of knowledge the students have been taught in the classrooms; and on the other hand, on the cultivation of capability of resolving complex engineering problems during the practice processes. Based on this concept, an innovative practice project financially supported by University of Shanghai for Science and Technology is explored as an example for carrying out a vivid engineering education.

The project entitled "Electrosprayed nanocomposites for better sustained-release of poorly water-soluble drug quercetin" was aimed to generate a new nanocomposite using electrospraying by four college students at their third grade year. The nanocomposites were in the forms of nanospheres, which could be utilized to manipulate the release behaviors of a traditional herb medicine, quercetin, also a poorly watersoluble drug. The formation of idea, the systematic implementation of electrospraying, and also the applications of final products are all a combination of a series of engineering issues. 


\section{ELECTROSPRAYING - A SIMPLE BUT Very COMPLICATED ENGINEERING PROCESS}

Electrospraying, akin to electrospinning, functions by dint of the fact that liquids can readily interact with electrical energy [2-9]. This energy is exploited to dry and solidify micro-fluid jets, causing solvent evaporation and producing micro-/nano- size products very rapidly (often on the order of 10-2 s). Provided favorable secondary interactions exist between the host polymeric matrix and the guest active ingredient, the physical state of the components in the solutions can be effectively propagated into the solid particles without phase separation, i.e. molecular composites. Certainly, as a result of the very fast drying process, the macroscale structure of the spray head is reproduced in the products on the microscale, such as core-shell structure from the coaxial electrospinning/electrospraying, Janus structure from side-byside electrospinning/electrospraying and tri-layer structure from tri-axial electrospinning [10-14].

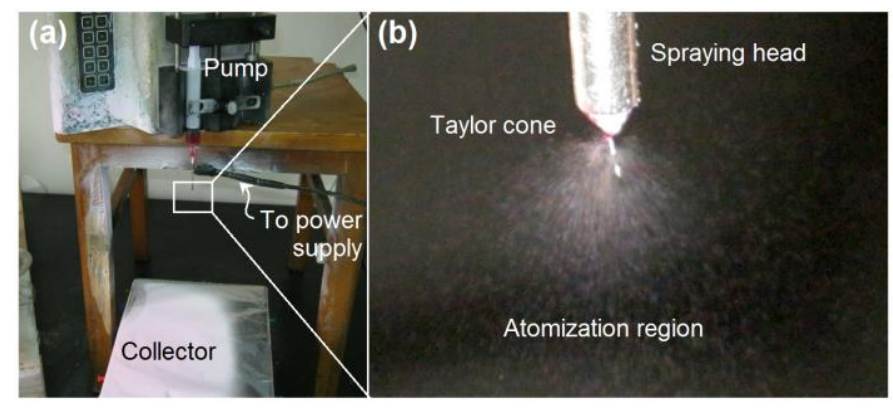

Fig. 1. A digital picture of the real electrospraying system (a) and the working process (b).

Electrospraying apparently is a very simple process. Shown in Figure 1, an electrospraying system consists of four components, i.e. a power supply to provide the high voltage; a syringe pump to quantitatively meter and drive the working fluid, a particle collector and a spray head (Figure 1a). An electrospraying process can be divided into four successive steps (i.e., the charge of working fluids; Taylor cone formation, droplet atomization, and the collection of final products) (Figure 1b). Although a simple and one-step straightforward "top-down" process, the mechanism of this blending electrospraying (and also electrospinning) is extremely complicated and no satisfied explanations up to date. Often a series of parameters (the working fluids' properties, the optimized working parameters for conducting electrospraying, and even the environmental conditions) interact with each other, making the resultant products difficult to be predicted. These parameters also reflect that electrospraying is a multidisciplinary phenomenon, such as electrostatic science, hydrodynamic engineering, rheology, polymer science and also the related applied material discipline. Even the solidification in the atomization region is a result of simultaneous phenomena, including solvent evaporation from electrohydrodynamic interfaces, polymer diffusion, and chain entanglement within droplets.

\section{COMPLICATED ISSUES FOR AN EXAMPLE APPLICATION - ELECTROSPRAYED NANOCOMPOSITES FOR DRUG SUSTAINED RELEASE}

Electrospraying has shown its usefulness in creating medicated micro/nanoparticles for various biomedical applications in the past two decades [2]. In view of applied raw materials, all pharmaceutical polymeric excipients, lipids, and even small molecules, such as urea, can be exploited for electrospraying; thus, electrospraying has a broader selection than electrospinning due to electrospinnability limitations [3]. In terms of controlled-release profiles of drugs, electrosprayed particles, just as medicated nanofibers, have been widely reported to provide all types of controlled-release profiles of drugs, such as immediate, sustained, targeted, dual, and multiple-phase releases [15-21]. Given the existence of numerous raw materials that can be treated using electrospraying and the demands of drug delivery systems with different types of controlled-release profiles of drugs, new electrospraying processes and insights on such advanced technologies should be accepted to promote the wide application of electrosprayed nanoparticles in biomedical fields. Particularly, these processes and insights may provide new solutions to key and important issues in the field of applied science, such as the effective delivery of poorly water-soluble drugs in pharmaceutics (Figure 2).

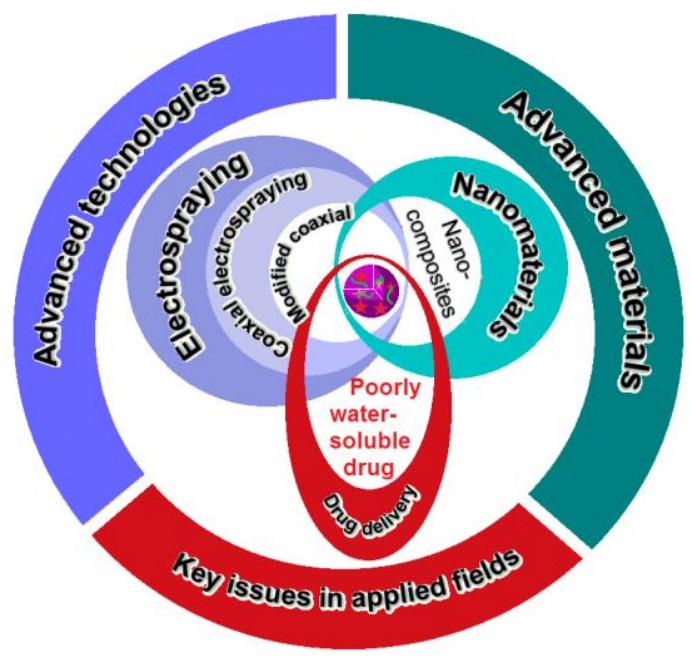

Fig. 2. Electrosprayed nanocomposites for drug sustained release is a complicated issue involving integrated multiple factors 


\section{COMPLICATED ISSUES FOR SYSTEMATIC IMPLEMENTATION OF ELECTROSPRAYING}

It seems a very easy thing to prepare solution for electrospraying, but the opposite is true. Numerous failures have resulted from lacking a suitable working solution. For preparing the drug-polymer co-dissolved solutions with enough drug concentrations and suitable physical-chemical property, a series of factors need to be considered systematically.

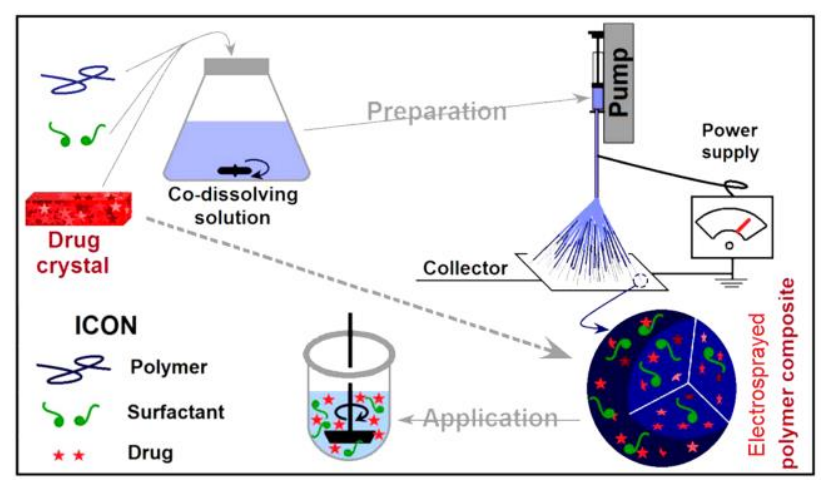

Fig. 3. The systematic implementation of electrospraying for creating polymer composites that provide drug sustained release profiles.

A typical procedure for systematic implementation of electrospraying is shown in Figure 3, in which a series of complex engineering problems should be encountered. Besides the multiple factors involved, these procedures may restrict each other and make the engineering problems even complicated. For example, the model drug quercetin is not soluble in the routine organic solvent such as methanol, ethanol, acetone and chloroform. It is soluble in $\mathrm{N}, \mathrm{N}$ dimethylacetamide (DMAc). However, DMAc has a high boiling point of $168 \mathrm{oC}$, which means that it is not suitable for preparing working solution because the polymer ethylcellulose (EC) can not be electrosprayed into solid nanoparticles directly. This complex problem could be resolved by trying a series of mixed solvents of ethanol and DMAc for meeting both enough quercetion concentration and solidifiable of EC.

A schematic diagram showing the factors that need to be taken into consideration for creating medicated polymer composites is shown in Figure 4. From the selection of raw materials (the model drug, the polymeric carrier's molecular weight and concentration in working fluid), to the processes (1fluid blending, coaxial, tri-axial and side-by-side), and to the key operational parameters (such as applied voltage, the nozzle-to-collector distance, the fluid flow rate), all are full of selections and optimizations. Only a suitable set of compatible selections can result in a fine final amorphous solid dispersion (ASD), i.e. molecular composites. Certainly, they are still some other complicated issues such as final applications of the electrosprayed particles. For examples, these particles can be easily exploited for providing sustained release, fast release and biphasic release [22-24]; the drugs can be both therapeutic ingredients and also agents for detection [25,26]; and the polymeric matrix can be water soluble, degradable or insoluble $[27,28]$.

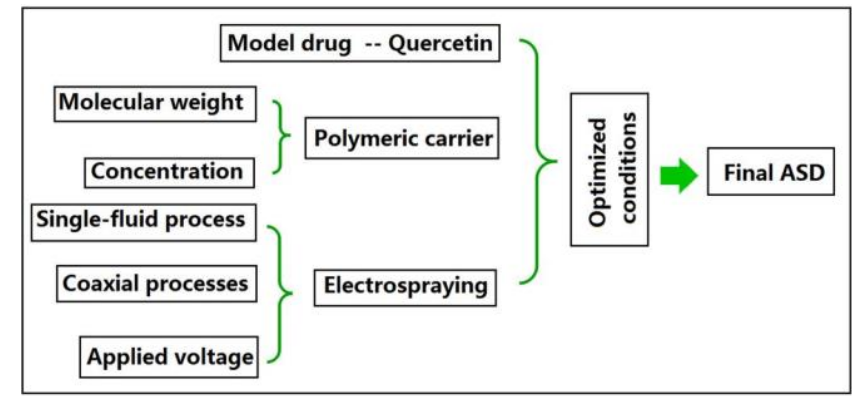

Fig. 4. A schematic diagram showing the factors that need to be taken into consideration for creating drug amorphous solid dispersion

As for a complicated engineering issue, it has at least three layers of meaning. First, the issue needs to be considered in a systematic manner. Second, the issue has a series of factors and these factors interact with each other. Third, the issue is a timedependent or step-by-step developing procedure. The advanced technologies such as electrospraying have all these concerns when they are exploited to prepare advanced materials. Thus, electrospraying can be exploited to teach the college students in two meanings. One is to teach them to implement this advanced method for creating novel materials. The other is to share with them the vivid lessons about finding and resolving complicated engineering problems.

\section{SUMMARY}

Several complex engineering issues have been refined from an innovation practice project for college students, whose title is "Electrosprayed nanocomposites for better sustained-release of poorly water-soluble drug - quercetin". These issues include a series of factors that would be encountered during the development of polymer composites for drug sustained release using electrospraying. The finding and resolving these issues should comprise a vivid teaching material for the students to learn how to foster their practice interests and to improve their innovative and engineering capabilities. On the other hand, the teaching of advanced technologies should include both their working processes and also the developments about thoughts on the related complex engineering issues.

\section{ACKNOWLEDGEMENT}

The financial supports from the following projects are appreciated: the Shanghai Education Science Research Project (C17058), the National Natural Science Foundation of China (No. 51373101), the 2017 Graduate curriculum reform project in USST, the Research Project on Vocational Education and Teaching in Guang-Xi Province (GXGZJG2016A028) and the College Student Innovation Project of USST (Nos. XJ2017286, SH2017189-190-191). 


\section{REFERENCES}

[1] Anonimity, https://en.wikipedia.org/wiki/Engineering, 21-September2017.

[2] X.Y. Li, Z.B. Zheng, D.G. Yu, X.K. Liu, Y.L. Qu, H.L. Li, Electrosprayed sperical ethylcellulose nanoparticles for an improved sustained-release profile of anticancer drug, Cellulose, 2017, DOI: 10.1007/s10570-017-1498-0

[3] Y.H. Wu, D.G. Yu, J.J. Li, Q. Wang, H.P. Li, X.Y. Li, Medicated multiple-component polymeric nanocomposites fabricated using electrospraying, Polym. Polym. Compos. 25 (2017) 57-62.

[4] U.E. Illangakoon, D.G. Yu, B.S. Ahmad, N.P. Chatterton, G.R. Williams, 5-Fluorouracil loaded Eudragit fibers prepared by electrospinning, Int. J. Pharm., 495 (2015) 895-902.

[5] G. Chen, Y. Xu, D.G. Yu, D.F. Zhang, N.P. Chatterton, K.N. White, Structure-tunable Janus fibers fabricated using spinnerets with varying port angles, Chem. Commun., 51 (2015) 4623-3626.

[6] G.Z. Yang, H.P. Li, J.H. Yang, J. Wan and D.G. Yu, Influence of working temperature on the formation of electrospun polymer nanofibers, Nanoscale Res. Lett., 12 (2017) 1-10.

[7] C. Sealy, New spin on drug-loaded composite fibers, Mater. Today, 19 (2016) 374-374

[8] C. Yang, D.G. Yu, D. Pan, X.K. Liu, X. Wang, S.W.A. Bligh, G.R Williams, Electrospun $\mathrm{pH}$-sensitive core-shell polymer nanocomposites fabricated using a tri-axial processes, Acta Biomater., 35 (2016) 77-86.

[9] Y.H. Wu, D.G. Yu, H.P. Li, X.Y. Wu, X.Y. Li, Medicated structural PVP/PEG composites fabricated using coaxial electrospraying, ePolymers, 17 (2017) 39-44.

[10] D.G. Yu, X.Y. Li, X. Wang, J.H. Yang, S.W.A. Bligh, G.R. Williams, Nanofibers fabricated using triaxial electrospraying as zero order drug delivery systems, ACS Appl. Mater. Interfaces, 7 (2015) 18891-18897.

[11] G.Z. Yang, J.J. Li, D.G. Yu, M.F. He, J.H. Yang, G.R. Williams, Nanosized sustained-release drug depots fabricated using modified triaxial electrospraying, Acta Biomater., 53 (2017) 233-241.

[12] D.G. Yu, K. White, N. Chatterton, Y. Li, L. Li, X. Wang, Structural lipid nanoparticles self-assembled from electrospun core-shell polymeric nanocomposites, RSC Adv., 5 (2015) 9462-9466.

[13] Q. Wang, H.P. Li, C. Yang, J.J. Li, D.G. Yu, Beads-on-a-string amorphous solid dispersion fabricated using a modified coaxial electrospraying, J. Control. Release, 259 (2017) e111-e112.

[14] D.G. Yu, J.J. Li, M. Zhang, G.R. Williams, High-quality Janus nanofibers prepared using three-fluid electrospraying, Chem. Commun., 53 (2017) 4542-4545.
[15] X. Wang, X.Y. Li, Y. Li, H. Zou, D.G. Yu, J.S. Cai, Electrospun acetaminophen-loaded cellulose acetate nanofibers fabricated using an epoxy-coated spinneret, e-polymers, 15 (2015) 311-315.

[16] Y.H. WU, C. Yang, X.Y. Li, J.Y. Zhu, D.G. Yu, Medicated nanofiber fabricated using $\mathrm{NaCl}$ solutions as shell fluids in a modified coaxial electrospraying, J. Nanomater., 2016 (2016) Article ID 8970213.

[17] Y.H. WU, H.P. Li, X.X. Shi, J. Wan, Y.F. Liu, D.G. Yu, Effective utilization of the electrostatic repulsion for improved alignment of electrospun nanofibers, J. Nanomater., 2016 (2016) Article ID2067383.

[18] Y.H. Wu, D.G. Yu, X.Y. Li, A.H. Diao, U.E. Illangakoon, G.R. Williams, Fast-dissolving sweet sedative nanofiber membranes, J. Mater. Sci., 50 (2015) 3604-3613

[19] Y. Xu, J.J. Li, D.G. Yu, G.R. Williams, J.H. Yang, X. Wang, Influence of the drug distribution in electrospun gliadin fibers on drug-release behavior, Eur. J. Pharm. Sci., 106 (2017) 422-430.

[20] H.F. Wen, C. Yang, D.G. Yu, X.Y. Li, D.F. Zhang, Electrospun zein nanoribbons for treatment of lead-contained wastewater, Chem. Eng. J., 290 (2016) 263-272.

[21] J.J. Li, C. Yang, H.P. Li, Q. Wang, D.G. Yu, Oral controlled release in accordance with drug adsorption biological rhythm provided by an electrospun structural amorphous solid dispersion, J. Control. Release, 259 (2017) e61-e62.

[22] D.G. Yu, H.P. Li, C. Yang, J.J. Li, Q. Wang, G.R. Williams. Doublepulsatile release core-shell fibers fabricated using modified tri-axial electrospraying, J. Control. Release, 259 (2017) e24-e25

[23] Q. Wang, D.G. Yu, S.Y. Zhou, C. Li, M. Zhao, Fabrication of amorphous electrospun medicated-nanocomposites using a Teflon-based concentric spinneret, e-Polymer, 2017, DOI: https://doi.org/10.1515/epoly-2017-0110

[24] D.G. Yu, C. Yang, M. Jin, G.R. Williams, H. Zou, X. Wang, S.W.A Bligh, Medicated Janus fibers fabricated using a Teflon-coated side-byside spinneret, Colloid. Surface. B, 138 (2016) 110-116.

[25] M. Jin, D.G. Yu, X. Wang, C.F.G.C. Geraldes, G.R. Williams, S.W.A Bligh, Electrospun contrast agent-loaded fibers for colon-targeted MRI, Adv. Healthcare Mater., 5 (2016) 977-985

[26] M. Jin, D.G. Yu, C.F.G.C. Geraldes, G.R. Williams, S.W.A. Bligh, Theranostic fibers for simultaneous imaging and drug delivery, Mol. Pharm., 13 (2016) 2457-2465.

[27] Q. Wang, D.G. Yu, L.L. Zhang, X.K. Liu, M. Zhao, Electrospun hypromellose-based hydrophilic composites for rapid dissolution of poorly water-soluble drug, Carbohydr. Polym. 174 (2017) 617-625.

[28] X. Wang, D.G. Yu, X.Y. Li, S.W.A. Bligh, G.R. Williams, Electrospun medicated shellac nanofibers for colon-targeted drug delivery, Int. J. Pharm., 490 (2015) 384-390. 